\title{
Observational constraints on general relativistic energy conditions, cosmic matter density and dark energy from X-ray clusters of galaxies and type-la supernovae
}

\author{
P. Schuecker ${ }^{1}$, R. R. Caldwell ${ }^{2}$, H. Böhringer ${ }^{1}$, C. A. Collins ${ }^{3}$, L. Guzzo ${ }^{4}$, and N. N. Weinberg ${ }^{5}$ \\ ${ }^{1}$ Max-Planck-Institut für extraterrestrische Physik, Giessenbachstraße 1, 85740 Garching, Germany \\ 2 Department of Physics \& Astronomy, Dartmouth College, Hanover, NH 03755, USA \\ 3 Astrophysics Research Institute, Liverpool John Moores University, Twelve Quays House, Egerton Wharf, \\ Birkenhead CH41 1LD, UK \\ ${ }^{4}$ INAF-Osservatorio di Brera, via Bianchi, 22055 Merate (LC), Italy \\ 5 California Institute of Technology, Mail Code 130-33, Pasadena, CA 91125, USA
}

Received 22 November 2002 / Accepted 6 February 2003

\begin{abstract}
New observational constraints on the cosmic matter density $\Omega_{\mathrm{m}}$ and an effectively redshift-independent equation of state parameter $w_{\mathrm{x}}$ of the dark energy are obtained while simultaneously testing the strong and null energy conditions of general relativity on macroscopic scales. The combination of REFLEX X-ray cluster and type-Ia supernova data shows that for a flat Universe the strong energy condition might presently be violated whereas the null energy condition seems to be fulfilled. This provides another observational argument for the present accelerated cosmic expansion and the absence of exotic physical phenomena related to a broken null energy condition. The marginalization of the likelihood distributions is performed in a manner to include a large fraction of the recently discussed possible systematic errors involved in the application of $\mathrm{X}$-ray clusters as cosmological probes. This yields for a flat Universe, $\Omega_{\mathrm{m}}=0.29_{-0.12}^{+0.08}$ and $w_{\mathrm{x}}=-0.95_{-0.35}^{+0.30}(1 \sigma$ errors without cosmic variance). The scatter in the different analyses indicates a quite robust result around $w_{\mathrm{x}}=-1$, leaving little room for the introduction of new energy components described by quintessence-like models or phantom energy. The most natural interpretation of the data is a positive cosmological constant with $w_{\mathrm{x}}=-1$ or something like it.
\end{abstract}

Key words. cosmology: cosmological parameters $-\mathrm{X}$ rays: galaxies: clusters

\section{Introduction}

Measurements of the cosmic microwave background (CMB) temperature anisotropies (e.g. Stompor et al. 2001; Netterfield et al. 2002; Pryke et al. 2002; Scott 2002; Sievers et al. 2002; Spergel et al. 2003), the redshift-distance relation of type-Ia supernovae (Riess et al. 1998; Perlmutter et al. 1999), the counts of galaxy clusters (e.g. Bahcall \& Fan 1998; Borgani et al. 2001; Reiprich \& Böhringer 2002), etc., suggest that we live in a dark-energy dominated Universe during a phase of accelerated cosmic expansion. Perhaps the simplest resolution is to resort to Einstein's cosmological constant $\Lambda$. Postulating a constant leaves many questions unanswered, however, relating to the nature of the particle physics vacuum and the (approximate) coincidence in the energy density of dark energy and dark matter today. For this finely-tuned constant the answer would seem to lie in the initial conditions. An alternative hypothesis is to consider a time-evolving dark energy, while assuming that any cosmological term is either zero or negligible. For a time-evolving inhomogeneous field (see e.g.

Send offprint requests to: P. Schuecker, e-mail: peters@mpe .mpg. de
Ratra \& Peebles 1988; Wetterich 1988; Caldwell et al. 1998; Caldwell 2002) the aim is to understand the coincidence in terms of dynamics.

A central rôle in these studies is assumed by the phenomenological ratio $w=p / \rho c^{2}$ between the pressure $p$ of the unknown energy component and its rest energy density $\rho$. In most investigations the parameter space of $w$ is restricted to $w \geq-1$ (exceptions are Caldwell 2002; Hannestad \& Mörtsell 2002; and Melchiorri et al. 2002) by assuming that the socalled null energy condition of general relativity should be fulfilled on macroscopic scales. However, for energy conditions no strict mathematical proofs exist hitherto, and their validity is not more than a conjecture. Therefore, the present investigation takes one step back, using $w$ itself to test the energy condition and finding the $w$ value which represents the observational data best.

Before we describe the test and its application to astronomical data we briefly review the significance of the energy conditions in cosmology relevant for the present work and their relation to $w$ (Sect. 2). The test is outlined in Sect. 3. In Sect. 4 the observational material used for the test is described. 
The results are presented in Sect. 5 and discussed in Sect. 6. In the following we define the Hubble constant in units of $h=H_{0} /\left(100 \mathrm{~km} \mathrm{~s}^{-1} \mathrm{Mpc}^{-1}\right)$.

\section{Energy conditions and cosmology}

Assumptions on energy conditions form the basis for the well-known singularity theorems (Hawking \& Ellis 1973), censorship theorems (e.g. Friedman et al. 1993) and no-hair theorems (e.g. Mayo \& Bekenstein 1996). Quantized fields violate all local point-wise energy conditions (Epstein et al. 1965). In the present investigation we are, however, concerned with observational studies on macroscopic scales relevant for cosmology where $\rho$ and $p$ are expected to behave classically. Normal matter in the form of baryons and non-baryons, or relativistic particles like photons and neutrinos satisfy all standard energy conditions. The two energy conditions discussed below are given in a simplified form (for more details see Wald 1984 and Barceló \& Visser 1999).

The strong energy condition (SEC): $\rho+3 p / c^{2} \geq 0$ and $\rho+$ $p / c^{2} \geq 0$, derived from the more general condition $R_{\mu \nu} v^{\mu} v^{v} \geq 0$, where $R_{\mu \nu}$ is the Ricci tensor for the geometry and $v^{\mu}$ a timelike vector. The simplified condition is valid for diagonalizable energy-momentum tensors which describe all observed fields with non-zero rest mass and all zero rest mass fields except some special cases (see Hawking \& Ellis 1973). The SEC ensures that gravity is always attractive. Certain singularity theorems (e.g., Hawking \& Penrose 1970) relevant for proving the existence of an initial singularity in the Universe need an attracting gravitational force and thus assume SEC. Violations of this condition as discussed in Visser (1997) allows phenomena like inflationary processes expected to take place in the very early Universe or a moderate late-time accelerated cosmic expansion as suggested by the combination of recent astronomical observations (see Sect. 1). Likewise, phenomena related to $\Lambda>0$ and an effective version of $\Lambda$ whose energy and spatial distribution evolve with time (quintessence: Ratra \& Peebles 1988; Wetterich 1988; Caldwell et al. 1998 etc.) are allowed consequences of the breaking of SEC - but not a prediction. However, a failure of SEC seems to have no severe consequences because the theoretical description of the relevant physical processes can still be provided in a canonical manner. Phenomenologically, violation of SEC means $w<$ $-1 / 3$ for a single energy component with density $\rho>0$. For $w \geq-1 / 3$, SEC is not violated and we have a decelerated cosmic expansion.

The null energy condition (NEC): $\rho+p / c^{2} \geq 0$, derived from the more general condition $G_{\mu \nu} k^{\mu} k^{\nu} \geq 0$, where $G_{\mu \nu}$ is the geometry-dependent Einstein tensor and $k^{\mu}$ a null vector (energy-momentum tensors as for SEC). Violations of this condition are recently studied theoretically in the context of macroscopic traversable wormholes (see averaged NEC: Flanagan \& Wald 1996; Barceló \& Visser 1999) and the holographic principle (see covariant entropy bound, McInnes 2002). The breaking of this criterion in a finite local region would have subtle consequences like the possibility for the creation of "time machines" (e.g. Morris et al. 1988). Violating the energy condition in the cosmological case is not as dangerous

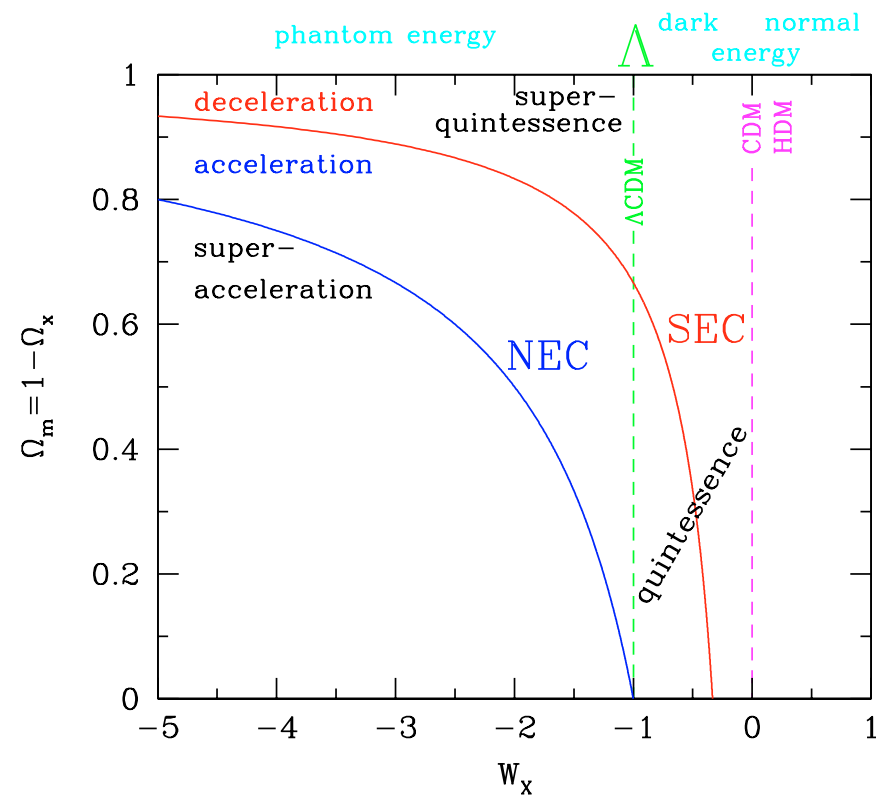

Fig. 1. The null energy condition (NEC) and the strong energy condition (SEC) for a flat FRW spacetime at redshift $z=0$ with negligible contributions from relativistic particles in the parameter space of the normalized cosmic density $\Omega_{\mathrm{m}}$ of baryonic and non-baryonic matter and the equation of state parameter $w_{\mathrm{x}}$ of a presently unknown energy component. The NEC and SEC curves are computed with Eqs. (7) and (8), respectively. The sector between the two curves gives accelerated growing scale factors where NEC is fulfilled but SEC is violated. In the sector below the NEC curve all energy conditions of general relativity are violated and the scale factor shows a super-accelerated increase. In the sector above SEC all energy conditions are fulfilled (especially also SEC) and no accelerated cosmic expansion is expected. The vertical line at $w_{\mathrm{x}}=-1$ mark models with Einstein's cosmological constant $(\Lambda>0$, especially Cold Dark Matter models $\Lambda \mathrm{CDM}$ ) and devides the parameter space into the quintessence-like sector $\left(-1<w_{\mathrm{x}}<0\right)$ based on dark energy and the super-quintessence sector $\left(w_{\mathrm{x}}<-1\right)$ based on phantom energy. For $0 \leq w_{\mathrm{x}} \leq 1$ ordinary energy might be expected in the form of Cold Dark Matter (CDM), Hot Dark Matter (HDM) etc.

(no threat to causality, no need to involve chronology protection, etc.), since one cannot isolate a chunk of the energy to power such exotic objects. Nevertheless, violation of NEC on cosmological scales could excite phenomena like super-acceleration of the cosmic scale factor (Caldwell 2002). Theoretically, violation of NEC would have profound consequences not only for cosmology because all point-wise energy conditions would be broken. It cannot be achieved with a canonical Lagrangian and Einstein gravity. Phenomenologically, violation of NEC means $w<-1$ for a single energy component with $\rho>0$. The sort of energy related to this state of a Friedmann-Robertson-Walker (FRW) spacetime is dubbed phantom energy and is described by superquintessence models (Caldwell 2002, see also Chiba et al. 2000). For $w \geq-1$ NEC is not violated, and the sort of energy is termed dark energy and is described by quintessence or super-quintessence models. 


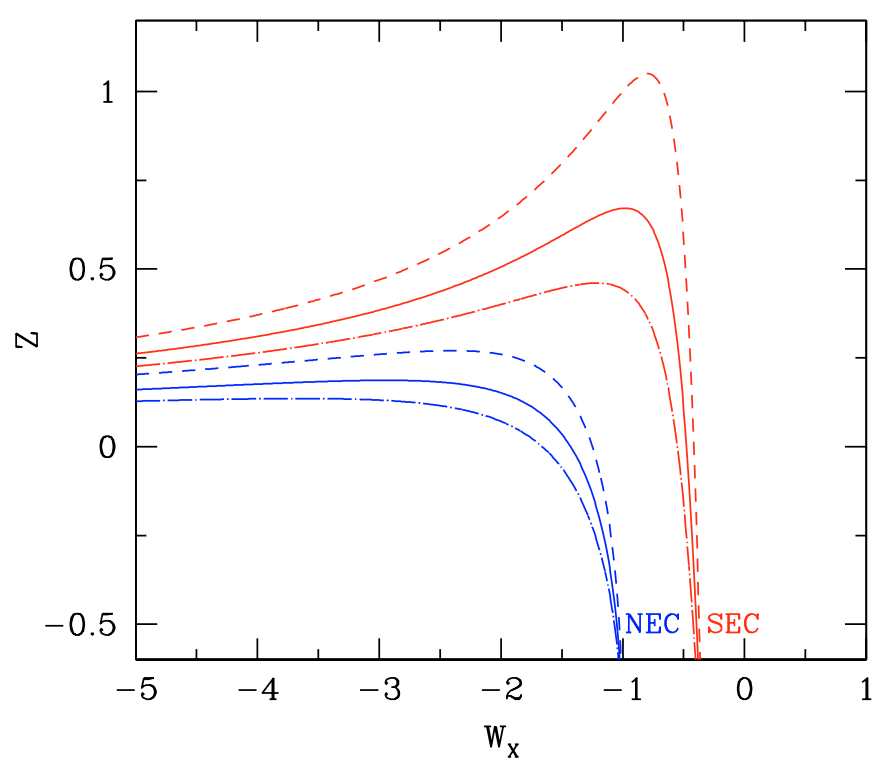

Fig. 2. The null energy condition (NEC) and the strong energy condition (SEC) for a flat FRW spacetime as a function of redshift $z$ and $w_{\mathrm{x}}$ with negligible contributions from relativistic particles computed with Eq. (11). Short dashed curves are computed with $\Omega_{\mathrm{m}}=0.2$, continuous curves with $\Omega_{\mathrm{m}}=0.3$, and dashed-dotted curves with $\Omega_{\mathrm{m}}=0.4$. Above the curves the respective energy conditions are fulfilled.

\section{A test of energy conditions on large scales}

The lack of verification of the energy conditions on macroscopic scales suggest that we should first test observationally the degree to which we can presently trust NEC and SEC. It will be seen that the test automatically yields those values of the present cosmic matter density and the equation-of-state parameter of the dark energy which describe the observational data best.

Our starting point is a FRW spacetime filled with a positive energy density of unknown nature which can be described as a perfect fluid $\left(p_{\mathrm{x}} \neq 0, \rho_{\mathrm{x}}>0, w_{\mathrm{x}}\right)$, with contributions from a pressureless non-baryonic and baryonic fluid $\left(p_{\mathrm{m}}=0, \rho_{\mathrm{m}}>0\right.$, $\left.w_{m}=0\right)$, and from relativistic particles like photons or neutri$\operatorname{nos}\left(p_{\mathrm{r}}>0, \rho_{\mathrm{r}}>0, w_{r}=1 / 3\right)$.

The different fluids "know about each other" through their common gravitational effects and through possible explicit couplings of one fluid to the others (a phenomenological treatment of a local energy transfer between two cosmic fluids can be found in Gromov et al. 2002). In the late Universe, a coupling between ordinary matter and the unknown energy component might remain (e.g. Amendola 2000), but the resulting effects are difficult to distinguish from predictions of general relativity plus a cosmological constant (Torres 2002). We thus regard the three fluids mentioned above as effectively independent substances over the redshift range covered by the astronomical objects used in our tests. Consequently, for each of the cosmic substances a local energy balance holds (e.g. Rindler 2001),

$\frac{\dot{\rho}}{\rho}+3(1+w) H=0$, derived from the twice-contracted Bianchi identity and Einstein's field equations for a perfect fluid. In (1), $H$ is the Hubble parameter and a dot denotes a derivative with respect to the cosmic time.

We are aware that these pre-assumptions are already quite specific compared to the usually targeted generality. However, our main focus is to learn more about the unknown energy component " $x$ " and the cosmic phenomena related to it. In this sense we proceed further and add all the energy and pressure sources to get the net equation for the pressumed multicomponent effective cosmic fluid. In this case, the NEC with its constraint on the passive gravitational mass density $\left(\rho+p / c^{2} \geq\right.$ $0)$ reads

$\rho_{\mathrm{x}}+\frac{p_{\mathrm{x}}}{c^{2}}+\rho_{\mathrm{m}}+\rho_{\mathrm{r}}+\frac{p_{\mathrm{r}}}{c^{2}} \geq 0$

or with the equation of state parameter $w_{\mathrm{x}}$ of the unknown fluid defined by $p_{\mathrm{x}}=w_{\mathrm{x}} \rho_{\mathrm{x}} c^{2}$, and $p_{\mathrm{r}}=\frac{1}{3} \rho_{\mathrm{r}} c^{2}$,

$\rho_{\mathrm{x}}+w_{\mathrm{x}} \rho_{\mathrm{x}}+\rho_{\mathrm{m}}+\rho_{\mathrm{r}}+\frac{1}{3} \rho_{\mathrm{r}} \geq 0$.

Using the normalized energy density of matter $\left(\Omega_{\mathrm{m}}\right)$, of relativistic particles $\left(\Omega_{\mathrm{r}}\right)$ and of the presently unknown energy component $\left(\Omega_{\mathrm{x}}\right)$, Eq. (3) can be recast into the inequality

$w_{\mathrm{x}} \geq-\frac{\Omega_{\mathrm{m}}+\frac{4}{3} \Omega_{\mathrm{r}}+\Omega_{\mathrm{x}}}{\Omega_{\mathrm{x}}}$,

NEC for $\Omega_{\mathrm{m}}, \Omega_{\mathrm{r}} \geq 0, \Omega_{\mathrm{x}}>0$. Similarily, the SEC with its additional constraint on the active gravitational mass density $\left(\rho+3 p / c^{2} \geq 0\right)$ corresponds to

$w_{\mathrm{x}} \geq-\frac{\Omega_{\mathrm{m}}+2 \Omega_{\mathrm{r}}+\Omega_{\mathrm{x}}}{3 \Omega_{\mathrm{x}}}$,

SEC for $\Omega_{\mathrm{m}}, \Omega_{\mathrm{r}} \geq 0 \Omega_{\mathrm{x}}>0$. Note that the inclusion of an unknown energy component in the form of a perfect fluid naturally extends earlier definitions of the SEC where cases with $\Lambda$-like energies were explicitly excluded. However, the present definition still follows the basic idea of the SEC because its validity guarantees that the active gravitational mass of a multicomponent cosmic fluid always leads to an attractive gravitational effect,

$\frac{\ddot{a}}{a}=-\frac{4 \pi G}{3} \sum_{i=x, m, r}\left(\rho_{i}+\frac{3 p_{i}}{c^{2}}\right) \leq 0$,

where $a=1 /(1+z)$ is the scale factor of the spacetime, $z$ the cosmological redshift, and where $\Lambda c^{2} / 3$ is replaced in the Friedmann-Lemaitre equation by the general term $8 \pi G \rho_{\mathrm{x}} / 3$.

For the more restrictive case of a spatially flat FRW geometry and a negligible contribution from relativistic particles (current estimates range from $\Omega_{\mathrm{r}}=0.001$ to maximal 0.05 , see Turner 2002) we have

$w_{\mathrm{x}} \geq-\frac{1}{1-\Omega_{\mathrm{m}}}$,

NEC for $\Omega_{\mathrm{m}} \geq 0$ and $\Omega_{\mathrm{m}}+\Omega_{\mathrm{x}}=1$, and

$w_{\mathrm{x}} \geq-\frac{1}{3\left(1-\Omega_{\mathrm{m}}\right)}$, 
SEC for $\Omega_{\mathrm{m}} \geq 0$ and $\Omega_{\mathrm{m}}+\Omega_{\mathrm{x}}=1$. Only for the unrealistic limit $\Omega_{\mathrm{m}} \rightarrow 0$ the NEC restriction (7) converges to the frequently adopted threshold $w_{\mathrm{x}}(\mathrm{min})=-1$. For $\Omega_{\mathrm{x}} \rightarrow 0$ and thus $\Omega_{\mathrm{m}} \rightarrow$ 1 one gets $w_{\mathrm{x}}(\min ) \rightarrow-\infty$ and both NEC and SEC are always fulfilled. The link between $w_{\mathrm{x}}$ and observable quantities like distances, volumes etc. is given by equations of the form

$$
\begin{aligned}
& {\left[\frac{H(z)}{H_{0}}\right]^{2}=\Omega_{\mathrm{x}} f\left(z, w_{\mathrm{x}}\right)+\Omega_{\mathrm{m}}(1+z)^{3}} \\
& +\Omega_{\mathrm{r}}(1+z)^{4}+\left(1-\Omega_{\mathrm{x}}-\Omega_{\mathrm{m}}-\Omega_{\mathrm{r}}\right)(1+z)^{2},
\end{aligned}
$$

which relate $H(z)$ to $w_{\mathrm{x}}, \Omega_{\mathrm{m}}, \Omega_{\mathrm{r}}$ and $\Omega_{\mathrm{x}}$. The redshiftdependency of the latter energy component in (9) can be obtained from the integration of (1) and is

$$
f\left(z, w_{\mathrm{x}}\right)=\exp \left\{3 \int_{0}^{z}\left[1+w_{\mathrm{x}}\left(z^{\prime}\right)\right] \mathrm{d} \ln \left(1+z^{\prime}\right)\right\}
$$

For simplicity we concentrate on a redshift-independent constant $w_{\mathrm{x}}$, so that $(10)$ leads to $f\left(z, w_{\mathrm{x}}\right)=(1+z)^{3\left(1+w_{\mathrm{x}}\right)}$. For $w_{\mathrm{x}}=-1$ we have $f=1$ and $\Omega_{\mathrm{x}}$ corresponds to the normalized cosmological constant $\Omega_{\Lambda}$ (Fig. 1).

For constant $w_{\mathrm{x}}$, the redshift-dependency of $(7,8)$ can be found by replacing the present matter density $\Omega_{\mathrm{m}}$ by $\Omega_{\mathrm{m}}(z)=$ $\Omega_{\mathrm{m}} /\left[\Omega_{\mathrm{m}}+\left(1-\Omega_{\mathrm{m}}\right)(1+z)^{3 w_{\mathrm{x}}}\right]$ leading to the condition

$$
1+z \geq\left[-\left(1+\frac{w_{\mathrm{x}}}{\gamma}\right) \frac{1-\Omega_{\mathrm{m}}}{\Omega_{\mathrm{m}}}\right]^{-\frac{1}{3 \omega_{\mathrm{x}}}}
$$

with $\gamma=1$ and $\frac{1}{3}$ for NEC and SEC, respectively, and $0<$ $\Omega_{\mathrm{m}}<1$ and $w_{\mathrm{x}}<-\gamma$. Above the redshift limit given in Eq. (11) the respective energy condition is fulfilled. Figure 2 suggests that for $z \gg 1$ (excluding cosmic epochs with e.g. dominating scalar fields like in a possible inflationary phase) and for reasonable present $\Omega_{\mathrm{m}}$ values, NEC and SEC are always fulfilled - independent of the value of $w_{\mathrm{x}}$. It is also seen that the frequently adopted lower NEC-threshold of $w_{\mathrm{x}}(\min )=-1$ is recovered at $z \ll 0$, that is, in the distant future. Note, however, that at extreme redshifts the constancy of $w_{\mathrm{x}}$ is expected to be a poor approximation, suggesting a failure of our considerations in these extreme $z$-ranges.

This simple discussion shows that for the evaluation of NEC and SEC in the given restrictive sense $(7,8)$ one has to measure $\Omega_{\mathrm{m}}$ and $w_{\mathrm{x}}$ (most importantly at the present epoch). In order to get robust observational constraints we utilize the complementarity of two approaches which have a different sensitivity on $w_{\mathrm{x}}$ and $\Omega_{\mathrm{m}}$. Recent supernova (SN) data constrain $w_{\mathrm{x}}$ and the cosmic matter density $\Omega_{\mathrm{m}}$, but the results are highly degenerated (Garnavich et al. 1998; Perlmutter et al. 1999). The degeneracy can be broken by using abundance measurements of a large sample of nearby X-ray clusters of galaxies which give a precise estimate of $\Omega_{\mathrm{m}}$ (Schuecker et al. 2003) almost independent of $w_{\mathrm{x}}$ (see Sect. 4.1). The results are compared with estimates obtained by a complementary approach which combines SN, CMB and other data (Sect. 6).

\section{Observational data}

\subsection{The X-ray cluster sample}

The ROSAT ESO Flux-Limited X-ray (REFLEX) sample used for the present investigations consists of the 452 X-ray brightest southern clusters of galaxies with redshifts mainly below $z=0.3$. They are extracted with a well-known selection function from the ROSAT All-Sky survey (Voges et al. 1999) and confirmed by extensive optical follow-up observations within a large ESO Key Programme (Böhringer et al. 1998; Guzzo et al. 1999). The clusters are located in an area of $4.24 \mathrm{sr}$ in the southern hemisphere with Declination $\leq 2.5 \mathrm{deg}$, excluding galactic latitudes $|b| \leq 20 \mathrm{deg}$ and some additional crowded fields like the Magellanic Clouds (Böhringer et al. 2001). The sample is expected to be at least $90 \%$ complete. With this sample the cluster X-ray luminosity function (Böhringer et al. 2002), the spatial cluster-cluster correlation function (Collins et al. 2000), its power spectrum (Schuecker et al. 2001), and the cosmic matter density (Schuecker et al. 2002, 2003) have been determined with unprecedented precision. The 426 REFLEX clusters used for the present abundance measurements have at least $10 \mathrm{X}$-ray source counts detected in the ROSAT energy band 0.5-2.0 keV, X-ray luminosities $L_{\mathrm{X}} \geq 2.5 \times 10^{42} h^{-2} \mathrm{erg} \mathrm{s}^{-1}$ and X-ray fluxes $S_{\mathrm{X}} \geq 3.0 \times$ $10^{-12} \mathrm{erg} \mathrm{s}^{-1} \mathrm{~cm}^{-2}$ in the energy band $0.1-2.4 \mathrm{keV}$.

The values of the cosmological parameters are estimated by comparing the observed redshift histogram of the clusters with model predictions. Note that the variances of the cosmological parameters shown here are larger compared to those obtained with the combined analysis of both the redshift histogram and the fluctuation power spectrum as used in Schuecker et al. (2003). A brief description of the model fits of the redshift histograms is given below. More details especially the values of important model parameters can be found in Schuecker et al. (2002, 2003). Deviations from the assumed values introduce possible systematic errors leading to a comparatively large scatter of, e.g., the measured $\sigma_{8}$ values (see Pierpaoli et al. 2002), where $\sigma_{8}$ gives the standard deviation of the matter density fluctuations in spheres with a comoving radius of $8 h^{-1} \mathrm{Mpc}$. In the present investigation we take these systematic errors into account by marginalizing the cluster likelihood distributions over a large $\sigma_{8}$ range (see below).

The number of clusters expected under REFLEX conditions at a specific redshift is given by the integral over the mass function where the lower mass limit is a function of redshift, flux-limit, cosmology etc. The mass limit is directly related to an X-ray luminosity via the mass/X-ray luminosity relation of galaxy clusters. We use an empirical estimate given in Reiprich \& Böhringer (2002). The resulting mass limit is transformed into the mass system defined in Jenkins et al. (2001) so that the corresponding mass function can be integrated to give the expected average number of clusters.

The computation of the X-ray luminosities takes into account the systematic underestimation of the observed unabsorbed X-ray fluxes of REFLEX clusters relative to the total fluxes (10\%, see Böhringer et al. 2002) and the cosmic $K$-corrections obtained from a refined Raymond-Smith code 

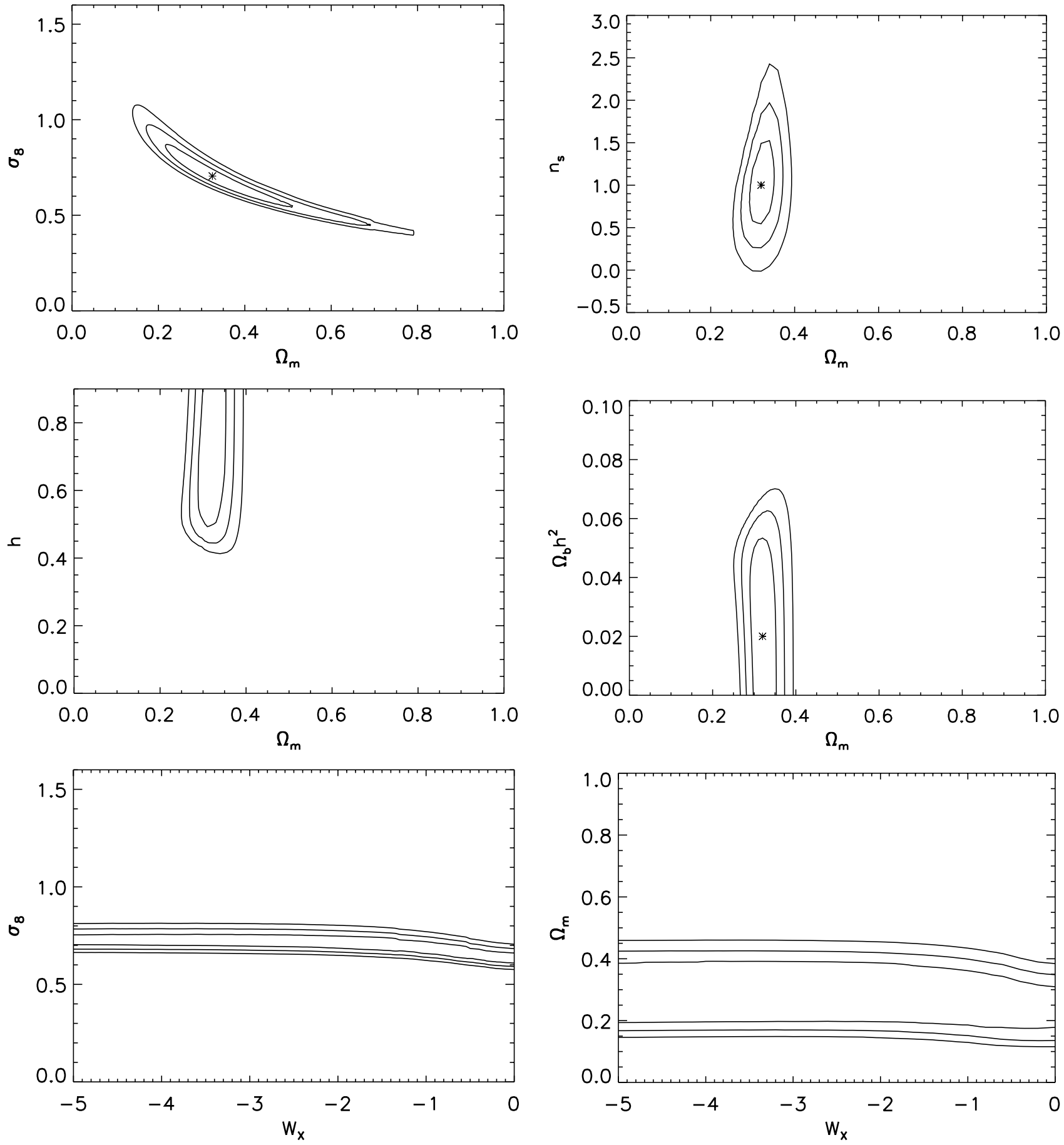

Fig. 3. Likelihood contours (1-3 $\sigma$ levels for two degrees of freedom) for various cosmological parameters obtained with the abundances of the REFLEX clusters. Note that the lower right panel includes marginalization over $\sigma_{8}=[0.70,0.95]$. The parameter priors of each diagram and the marginalization range are given in the main text.

(Böhringer et al. 2000). For the transformation of the cluster masses defined at different overdensity radii the Navarro et al. (1997) mass density profile is used with a redshift and massindependent concentration parameter of $c=5$. Deviations from this value in the range $4 \leq c \leq 6$ have effects below a few percent and are neglected (Schuecker et al. 2003). We thus assume that the REFLEX clusters do not show any significant evolution up to $z=0.3$ as suggested by the redshift-independent distribution of the comoving number densities of the REFLEX clusters (Schuecker et al. 2001, see also the discussion in Rosati et al. 2002). The integration of the mass function includes a convolution which takes into account the intrinsic scatter of the mass/X-ray luminosity relation, and the random flux (luminosity) errors of the REFLEX clusters as given in Böhringer et al. (2001). In the present investigation we use an effective scatter with a formal value of $25 \%$ which includes the contributions from flux errors (10\%) and intrinsic scatter (20\%). The intrinsic scatter is in reasonable agreement with the 
Reiprich \& Böhringer (2002) mass/X-ray luminosity relation if one takes into account that realistic mass errors are expected to be a factor 1.5 larger than the formal errors given in Reiprich \& Böhringer (see Schuecker et al. 2003 for a more detailed discussion).

The computation of the theoretical mass function assumes a matter power spectrum and a model for the critical density contrast which defines the virial cluster mass. For the matter power spectrum a Cold Dark Matter transfer function with a given contribution of baryons is assumed (see Eisenstein \& $\mathrm{Hu}$ 1998). No $w_{\mathrm{x}}$-dependent corrections of the transfer functions as given in Ma et al. (1999) were applied because of the comparatively small redshift range $(z<0.3)$ and scale range $\left(<1.5 h^{-1} \mathrm{Gpc}\right)$ covered by the given X-ray cluster sample. For the determination of the $w_{\mathrm{x}}$ (and $\Omega_{\mathrm{m}}$ ) dependent critical density contrasts we follow the formalism of Wang \& Steinhardt (1998) and integrate numerically the relevant ordinary differential equations describing the collapse of a spherical overdensity in an expanding Universe with $w_{\mathrm{x}} \neq-1$. We found that the $\zeta$ function introduced in Wang \& Steinhardt (their Eq. (A11)) used to compute the average critical density contrast between cluster and background should be replaced by

$\zeta=5.4\left[\Omega_{\mathrm{m}}\left(z_{\mathrm{ta}}\right)\right]^{-0.666}+0.11\left[\Omega_{\mathrm{m}}\left(z_{\mathrm{ta}}\right)\right]^{-1.85}\left(1-w_{\mathrm{x}}\right)^{-1.5}$,

giving a good approximation (better than 5\%) for the range $-5<w_{\mathrm{x}}<0$ at turn-around $\left(z_{\mathrm{ta}}\right)$. Equation (12) deviates from the corresponding equation obtained for the smaller range $-1<w_{\mathrm{x}}<0$ given in Wang \& Steinhardt by about $5 \%$ and is larger by a factor 1.3 at $w_{\mathrm{x}}=-5$ (for $\Omega_{\mathrm{m}}=0.3$ ). For completeness we also give the equivalent linear overdensity of a virialized spherical shell,

$\delta_{c}=1.686 \Omega_{\mathrm{m}}^{0.037\left(1-w_{\mathrm{x}}\right)^{-2.7}}$,

which is needed when the Press-Schechter and the ShethTormen mass functions are used. In the present case we use the Jenkins et al. mass function because of its higher precision where $\delta_{\mathrm{c}}$ is not needed. Nevertheless, for $-5<w<-1$ there is almost no change in $\delta_{\mathrm{c}}$ except for ridiculously small values of $\Omega_{\mathrm{m}}$.

The comparison of expected and observed cluster abundances assumes a Gaussian likelihood distribution. For large sizes of the count cells (as used here) this assumption is justified at a high level of statistical significance by the measurements of Schuecker et al. (2002, 2003).

The likelihood contours shown in Fig. 3 illustrate the sensitivity of the REFLEX sample to specific cosmological parameters for a flat cosmic geometry. The set of default parameter values are: $h=0.70$ (Freedman et al. 2001), $\sigma_{8}=0.711$ (normalization of the matter power spectrum, see Schuecker et al. 2003; Allen et al. 2002), $\Omega_{\mathrm{m}}=0.341$ (see Schuecker et al. 2003), $n_{\mathrm{S}}=1.0$ (spectral index of initial scalar fluctuations, see the recent CMB measurements given in Sect. 1), $\Omega_{\mathrm{b}} h^{2}=0.022$ (baryon density, see CMB), $w_{\mathrm{x}}=-1$. Each panel in Fig. 3 shows the $1-3 \sigma$ likelihood contours for two parameters whereby the remaining parameter values are fixed by the default values given above.

Notice that the cosmological parameters $\sigma_{8}$ and especially $\Omega_{\mathrm{m}}$ can be obtained with nearby cluster samples almost independent of the value of $w_{\mathrm{x}}$, as seen in the lower panels of Fig. 3. $\Omega_{\mathrm{m}}$ measurements based on the abundance of nearby clusters thus appear quite stable against the presence of ordinary or more exotic $\Lambda$-like energies.

The lower right panel of Fig. 3 gives the final $\left(w_{\mathrm{x}}, \Omega_{\mathrm{m}}\right)$ likelihood values which will be used for the combination with the SN data (see Sect. 5). The likelihood distribution is obtained with $h=0.70, n_{\mathrm{S}}=1, \Omega_{\mathrm{b}} h^{2}=0.022$ and after marginalization over the quite large $\sigma_{8}$ range of [0.70, 0.95]. This interval covers most of the $\sigma_{8}$ values obtained recently with different samples, model assumptions, and methods (weak lensing, optical clusters, X-ray cluster temperature and luminosity functions, power spectrum, Sunyaev-Zel'dovich effect power spectrum, galaxy clustering etc.) as summarized in, e.g., Pierpaoli et al. (2002). Compared to this marginalization range, the relatively small systematics introduced by the uncertainties in the values of $n_{\mathrm{S}}, h$, and $\Omega_{\mathrm{b}} h^{2}$ are neglected (see Fig. 3, see also Table 1 in Schuecker et al. 2003).

\subsection{The type-la supernova samples}

The two SNe Ia samples described in Riess et al. (1998) and Perlmutter et al. (1999) are used for the present investigations. The distance moduli of the $\mathrm{SNe}$ are determined with spectral and photometric observations. The host-galaxy subtracted SN peak magnitudes are corrected by the two teams for the cosmic $K$-effect, absorption in the Galaxy and host galaxy assuming a standard galactic absorption law and no colour evolution (Perlmutter et al. did not correct for absorption in the host galaxy but made some additional checks and rejected obviously reddened $\mathrm{SNe}$ from the cosmological tests), time dilation in the light curve, and the shape of the light curve. There is a distinct difference in the treatment of the latter correction in the two samples which complicates a direct comparison between the two data sets. The difference is, however, not relevant for cosmological applications.

Corrections of the change in the peak absolute luminosity are performed for each SN Ia in the Riess et al. sample with the $\Delta m_{15}$ method of Phillips (1993), Hamuy et al. (1995), and Phillips et al. (1999), and with the Multi-Color Light Curve Shape (MCLS) method of Riess et al. (1998). A simple renormalization of the observed apparent peak magnitude is performed for each SN Ia in the Perlmutter et al. sample using the stretch factor introduced in Perlmutter et al. (1995, 1997).

The Riess et al. sample consists of 27 nearby SNe Ia $(z<$ $0.2), 10$ High $-z$ SNe Ia $(0.30 \leq z \leq 0.97)$ and 6 High- $z$ Snapshot SNe Ia $(0.16 \leq z \leq 0.83)$. The resulting catalogue used here gives the redshifts and the distance moduli as obtained with the $\Delta m_{15}$ and with the MCLS methods. The magnitudes are corrected for the various effects mentioned above. The errors of the distance moduli and cosmological redshifts of the $\mathrm{SNe}$ are used for weighting.

The Perlmutter et al. sample consists of 38 SCP SNe Ia $(0.172 \leq z \leq 0.830)$ and 16 Calán/Tololo SNe Ia $(0.014 \leq z \leq$ $0.101)$. The sample is the same as used by Perlmutter et al. for their Primary Fit (C-fit). Note that this sample excludes $6 \mathrm{SNe}$ from the original sample of $60 \mathrm{SNe}$ (2 residual outliers, 

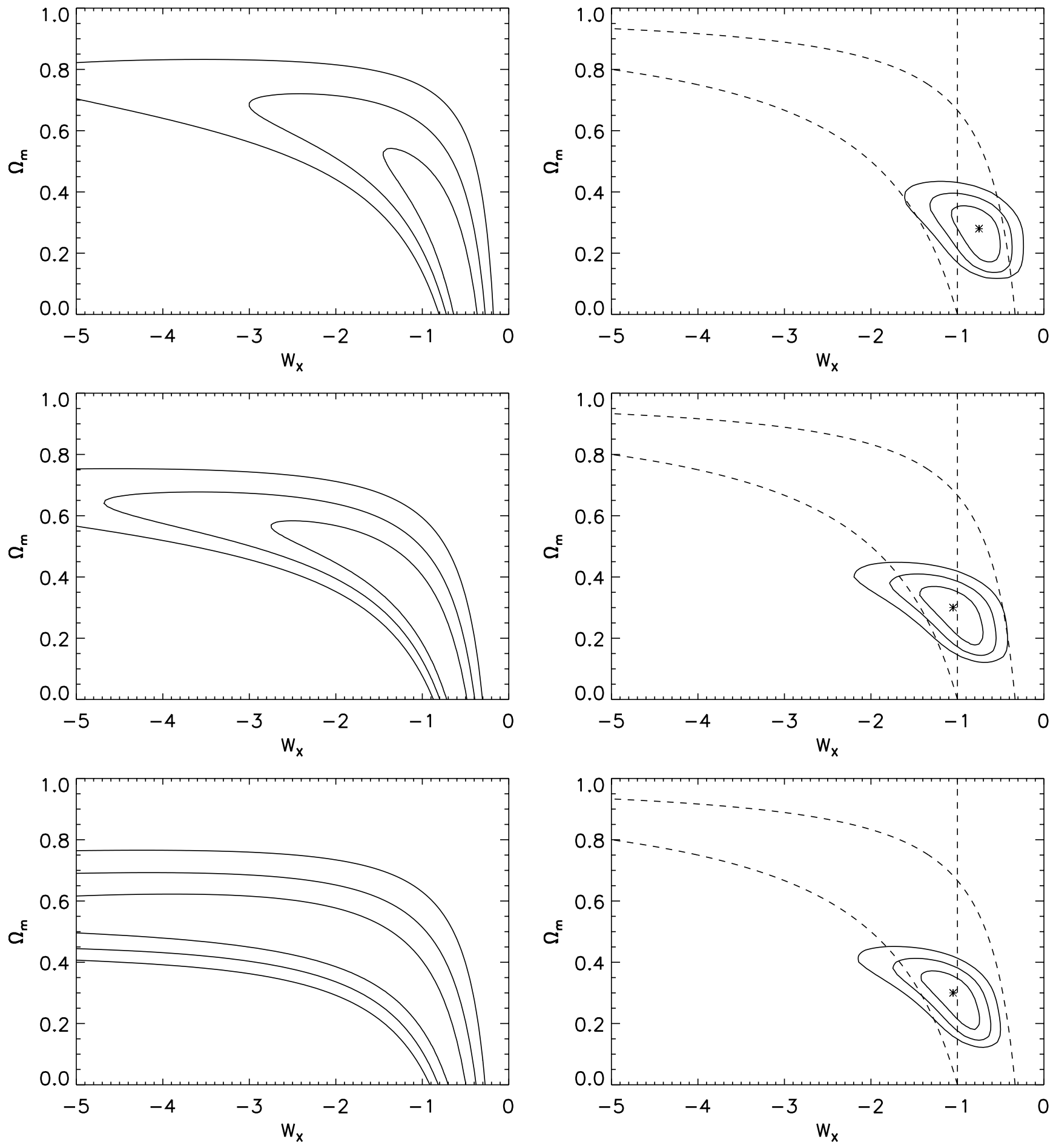

Fig. 4. Likelihood contours (1-3 $\sigma$ levels for two degrees of freedom) obtained with SNe Ia only (left panels) and with SNe Ia plus REFLEX X-ray clusters (right panels) marginalized over $\sigma_{8}=[0.70,0.95]$. The SN results of the first two rows are based on the Riess et al. (1998) sample (MCLS corrections upper row, $\Delta m_{15}$ corrections middle row), whereas the data in the lower row are based on the Perlmutter et al. (1999) sample. Vertical dashed lines at $w_{\mathrm{x}}=-1$ represent models with Einstein's cosmological constant. Curved dashed lines devide the parameter spaces into sectors where SEC is valid indicating a decelerated cosmic expansion (above the upper dashed curves), sectors where SEC is violated but NEC is fulfilled indicating an accelerated cosmic expansion (between the dashed curves), and sectors where NEC (and thus SEC) are violated indicating a super-accelerated cosmic expansion (below the lower dashed curves).

2 stretch outliers, 2 likely reddened $\mathrm{SNe})$. The resulting catalogue used here gives the SN redshifts, the effective (corrected) peak apparent magnitudes and the total uncertainty of the magnitudes. The latter quantities already include the uncertainties related to the expected errors of the cosmological redshifts of the SNe.

Note that the 16 low redshift Calán/Tololo SNe Ia and two distant $\mathrm{SNe}$ are members of both $\mathrm{SN}$ samples. Therefore, 
we cannot regard the Riess et al. and the Perlmutter et al. SN sample as statistically independent.

The present SN likelihood analyses assume Gaussian likelihood functions as verified in Riess et al. and Perlmutter et al. With the corrected peak magnitudes and redshifts of the $\mathrm{SNe}$ it is straightforward to compare observed and model magnitudes assuming different values of $w_{\mathrm{x}}$ and $\Omega_{\mathrm{m}}$ (including marginalization over method-specific quantities as in Riess et al. and Perlmutter et al.). The results are shown for the different samples and light curve corrections in the left panels of Fig. 4.

\section{Combined constraints from X-ray clusters and $\mathrm{SNe}$-la}

The combination of the likelihood distributions obtained with the X-ray clusters and SNe makes the realistic assumption that both samples are statistically independent so that the cluster and SN likelihoods can be point-wise multiplicated. The resulting joint likelihood distributions of the constraints on $w_{\mathrm{x}}$ and $\Omega_{\mathrm{m}}$ obtained for the three SN samples combined with the REFLEX results are shown in the right panels of Fig. 4 (see also Table 1). As discussed in Sect. 3, vertical dashed lines at $w_{\mathrm{x}}=-1$ represent the case of a cosmological constant and devide the parameter spaces into the dark energy sectors with $-1<w_{\mathrm{x}}<0$ and the phantom energy sectors with $w_{\mathrm{x}}<-1$. The curved dashed lines are computed with Eqs. (7) and (8) and give the deviding lines for NEC (lower dashed curves) and SEC (upper dashed curves).

Figure 4 shows that for all three combinations of X-ray cluster data obtained with the marginalization interval $\sigma_{8}=$ $[0.70,0.95]$ and SN data the centroids of the joint likelihood distributions range between $-1.05 \leq w_{\mathrm{x}} \leq-0.75$ and $0.28 \leq$ $\Omega_{\mathrm{m}} \leq 0.30$ (see also Table 1 ).

Figure 5 illustrates the stability of the results by showing the combined likelihood distributions for the $\sigma_{8}$ marginalization intervals [0.70, 0.75] (first row), [0.70, 0.80] (second row), $[0.70,0.85]$ (third row), $[0.70,0.90]$ (fourth row). These computations thus illustrate the effects of increasing systematic errors without identifying the exact sources of the systematics. In all cases the likelihood distributions have maxima at $w_{\mathrm{x}}$ values between -1.10 and -0.75 , and $\Omega_{\mathrm{m}}$ values between 0.28 and 0.32 .

The differences seen in Figs. 4 and 5 are attributed to the different methods used to correct the SN peak magnitudes, SN sample-to-sample variations, and different random plus systematic errors in the reduction of the cluster data. In all cases the centroids clearly fall between the NEC and SEC lines. Formal averages over the mean values and their $1 \sigma$ errors (without cosmic variance) obtained with the largest marginalization range of $\sigma_{8}=[0.70,0.95]$ give the final (most conservative) results,

$\Omega_{\mathrm{m}}=0.29_{-0.12}^{+0.08}, \quad w_{\mathrm{x}}=-0.95_{-0.35}^{+0.30}$.

Due to the statistical dependencies of the individual SN samples, (14) gives the mean errors obtained with the individual cluster-SN likelihood combinations, and not the errors of the averaged $\Omega_{\mathrm{m}}$ and $w_{\mathrm{x}}$ values.
Table 1. Constraints on $\Omega_{\mathrm{m}}$ and $w_{\mathrm{x}}$, and their $1 \sigma$ errors obtained with $\mathrm{SNe}$ plus X-ray clusters of galaxies after marginalization over $\sigma_{8}=[0.70,0.95]$ assuming a flat geometry. SNe: supernova sample, LCC: light curve correction, MCLS: multi-color light curve shape correction, $\Delta m_{15}$ correction, $s$ : stretch factor correction.

\begin{tabular}{lccc}
\hline \hline SNe & LCC & $\Omega_{\mathrm{m}}$ & $w_{\mathrm{x}}$ \\
\hline Riess & MCLS & $0.28_{-0.11}^{+0.09}$ & $-0.75_{-0.35}^{+0.25}$ \\
Riess & $\Delta m_{15}$ & $0.30_{-0.12}^{+0.08}$ & $-1.05_{-0.35}^{+0.35}$ \\
Perlmutter & $s$ & $0.30_{-0.12}^{+0.08}$ & $-1.05_{-0.35}^{+0.30}$ \\
\hline Average & & $0.29_{-0.12}^{+0.08}$ & $-0.95_{-0.35}^{+0.30}$ \\
\hline
\end{tabular}

\section{Summary and conclusions}

The null energy condition (NEC) and the strong energy condition (SEC) of general relativity are tested and give observational constraints on cosmic phenomena like quintessence, super-quintessence, and Einstein's cosmological constant. In order to test NEC and SEC on cosmic scales we assume a flat geometry and that baryonic matter, non-baryonic matter, relativistic matter and $\Lambda$-like matter can be regarded as independent perfect fluids. The resulting inequalities (Eqs. (7) and (8)) reproduce the frequently used threshold $w_{\mathrm{x}}=-1$ only in the unrealistic limit of a zero density of ordinary matter. Note that the usually adopted threshold is based on the unknown component $\Omega_{\mathrm{x}}$ only, which is not enough to cover the large diversity of phenomena caused by a mixture of different cosmic fluids expected to fill the present Universe (Fig. 1).

In this sense simple cosmological tests of NEC and SEC are formulated and applied to the presently largest homogeneously selected sample of X-ray cluster of galaxies (REFLEX). The most important advantage of X-ray clusters as summarized in Borgani \& Guzzo (2001) is that in contrast to optically selected clusters, their selection function (e.g. the sample volume, see Böhringer et al. 2002) and the relation between cluster X-ray luminosity and total cluster mass (see Reiprich \& Böhringer 2002) is well-known without the need of extensive numerical simulations. Moreover, the relation between the presence of a cluster in X-rays and a peak in the underlying cosmic mass distribution (cluster biasing) can be derived from first principles (e.g. Kaiser 1994; Matarrese et al. 1997; Moscardini et al. 2000). Therefore, the abundances of the nearby clusters are quite sensitive to $\Omega_{\mathrm{m}}$ and almost independent of $w_{\mathrm{x}}$, which is optimal to break the degeneracy between $\Omega_{\mathrm{m}}$ and $w_{\mathrm{x}}$ shown by the SN data.

Recently, detailed studies showed that for the application of $\mathrm{X}$-ray clusters as cosmological probes several systematic errors could quite strongly affect the final results (H. Böhringer et al., in preparation; Pierpaoli et al. 2002; Reiprich \& Böhringer 2002; Schuecker et al. 2003). This is reflected in a large scatter of $\sigma_{8}$ values published recently by different groups using different samples and methods and by comparing X-ray cluster results with results obtained with, e.g., weak lensing, optical clusters, X-ray cluster temperature and luminosity functions, power spectrum, Sunyaev-Zel'dovich effect power spectrum, 

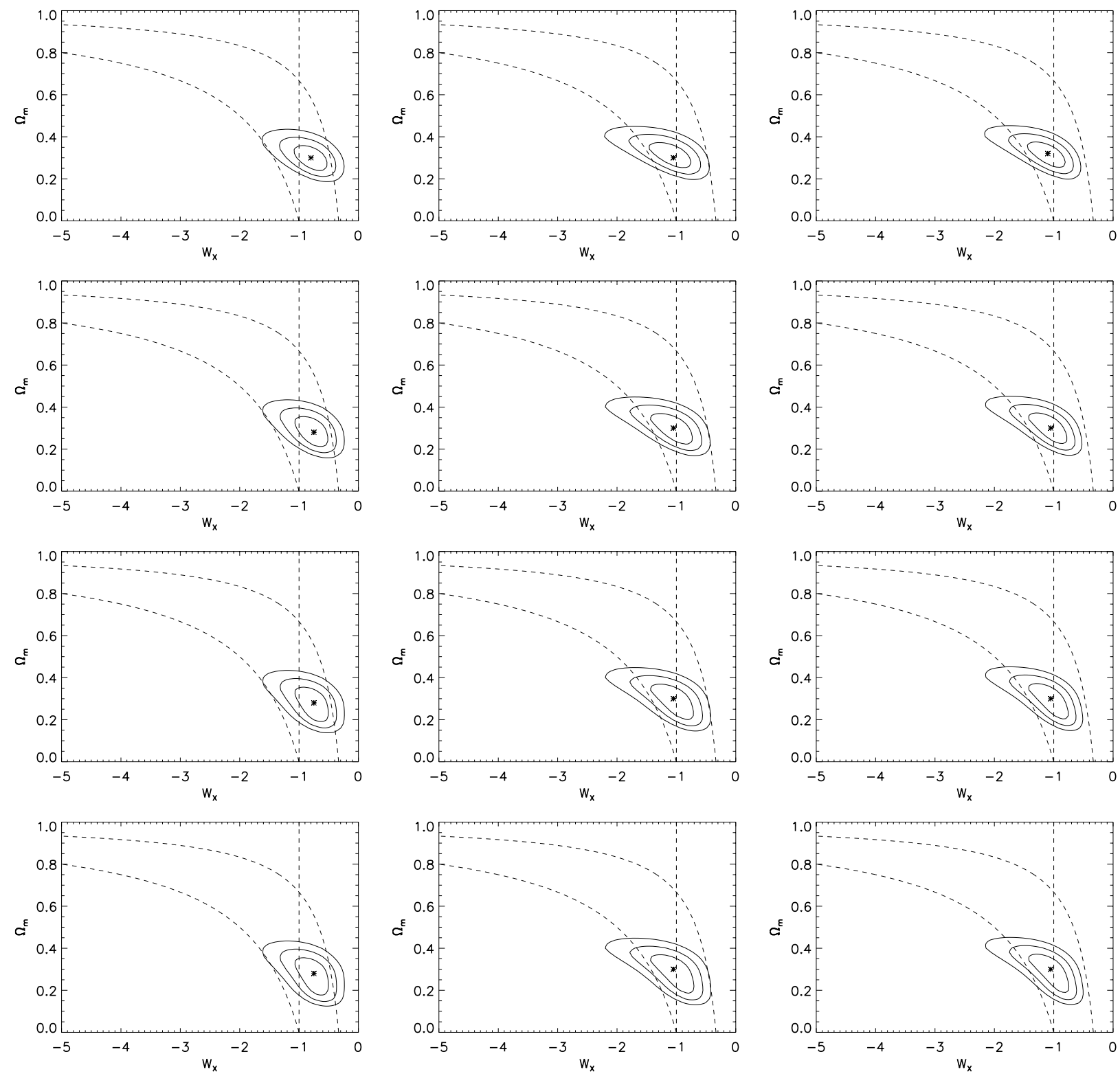

Fig. 5. Likelihood contours (1-3 $\sigma$ levels for two degrees of freedom) obtained with SNe Ia plus REFLEX X-ray clusters illustrating the stability of the SEC and NEC tests by changing the $\sigma_{8}$ ranges for marginalization. First column: Riess et al. (MCLS corrections) plus REFLEX. Second column: Riess et al. ( $\Delta m_{15}$ corrections) plus REFLEX. Third column: Perlmutter et al. plus REFLEX. First row: $\sigma_{8}=[0.70,0.75]$. Second row: $\sigma_{8}=[0.70,0.80]$, Third row: $\sigma_{8}=[0.70,0.85]$, Fourth row: $\sigma_{8}=[0.70,0.90]$.

galaxy clustering etc. - although some kind of convergence to specific $\Omega_{\mathrm{m}}$ and $\sigma_{8}$ values emerges. The present investigation takes this imprecise knowledge into account by a marginalization over a quite large range of $\sigma_{8}$ values $[0.70,0.95]$ which includes about $75 \%$ of the values obtained within the past two years. In comparison to this range, additional marginalization over our imprecise knowledge of cosmological parameters in the ranges $0.64 \leq h \leq 0.80,0.018 \leq \Omega_{\mathrm{b}} h^{2} \leq 0.026$, and $0.8 \leq n_{\mathrm{S}} \leq 1.2$ was analysed and shown to be of secondary importance and is thus neglected in the final results presented here.

It is quite important to note that the final $\Omega_{\mathrm{m}}$ and $w_{\mathrm{x}}$ values obtained in the present investigation are almost independent on the assumed marginalization ranges. Different choices in $\sigma_{8}$ yield changes in the final results always smaller than about $7 \%$ in both $\Omega_{\mathrm{m}}$ and $w_{\mathrm{x}}$. An increased marginalization range soley increases the final error bars and leaves the centroid values almost unchanged. We attribute the robustness of the test to the complementarity of SN-Ia and X-ray cluster data. Future investigations of $w_{\mathrm{x}}$ will clearly benefit from this complementarity.

The present analysis neglects the $w_{\mathrm{x}}$-dependency of both the shape, and the amplitude growth of the power spectrum of the matter density fluctuations. This is justified by the limited spatial scale and redshift ranges covered by the REFLEX cluster sample. Future cluster samples will hardly reach $5 h^{-1} \mathrm{Gpc}$ scales or so where linear theory $w_{\mathrm{x}}$-dependent effects on the shape of the power spectrum are formally expected. However, 
$w_{\mathrm{x}}$ can change the amplitude of the power spectrum by a factor of about two between redshift zero and $z=1$ which could be measured if the cluster X-ray luminosity/mass conversion and the effective biasing parameter of the sample could be computed with high enough accuracy. Deep and wide X-ray cluster samples could thus use in addition to the mean cluster abundance as the traditional cluster criterion (as described in Haiman et al. 2001) another quite strong $w_{\mathrm{x}}$-dependent criterion related to the fluctuations of the cluster counts around their mean abundance.

In the present investigation the joint likelihood distributions of the combination of X-ray cluster and SN data are computed as a function of the present-day $\Omega_{\mathrm{m}}$ and $w_{\mathrm{x}}$ values. NEC and SEC are thus effectively tested at redshift zero.

The combined data fall with about $1.5-4 \sigma$ statistical significance (depending on the SN sample and light curve correction) below the SEC threshold. The SEC can thus tendatively be regarded as broken on cosmic scales at $z=0$. A similar breaking of SEC was found by Visser (1997) under more general assumptions for the time between the epoch of galaxy formation and the present where he compares the relation between the age of the Universe and the age of the oldest observed stars. However, our theoretical expectations suggest that the broken SEC state should not hold for redshifts $z>(0.28-$ 1.20) (Eq. (11) and the $1 \sigma$ error corridor given in Eq. (14)), in contrast to the larger $z$ range implied by the analysis of Visser.

The data fall above the NEC threshold with about $1.5-3 \sigma$ statistical confidence, again depending on the SN sample and light-curve correction used. NEC can thus tendatively be regarded as fulfilled.

In every case tested sofar, the combined X-ray cluster and $\mathrm{SN}$ data obviously populate the sector between NEC validation and SEC violation and thus provide further observational evidence for an accelerated cosmic expansion at $z=0$.

The observational constraints obtained from the combination of X-ray cluster and SN data on $\Omega_{\mathrm{m}}$ are in good agreement with recent cluster data (e.g. Borgani et al. 2001; Allen et al. 2002; Schuecker et al. 2002, 2003; Pierpaoli et al. 2002), constraints from CMB data (see references given in Sect. 1, especially the WMAP result $\Omega_{m}=0.29 \pm 0.07,68 \%$ confidence, WMAP data only, Spergel et al. 2003), and galaxy data (Szalay et al. 2001; Lahav et al. 2002).

Our results on $w_{\mathrm{x}}$ are consistent with the constraints obtained from type-Ia supernovae (Garnavich et al. 1998; Perlmutter et al. 1999), with recent CMB data (e.g., Baccigalupi et al. 2002; Bond et al. 2002) and with the baryonic fraction in galaxy clusters (Ettori et al. 2003). The results obtained by the combined analysis of CMB and SN data of Hannestad \& Mörtsell (2002) and Melchiorri et al. (2002) yield the respective $95 \%$ confidence constraints $-2.68<w_{\mathrm{x}}<-0.78$ and $-1.62<w_{\mathrm{x}}<-0.74$ (see also Caldwell 2002), quite consistent but slightly larger than the results obtained with the $\mathrm{SN}$ and X-ray cluster data given here. Finally, the constraint $w_{\mathrm{x}} \leq-0.78$ is obtained with $95 \%$ confidence from the combination of WMAP, SN, 2dFGRS and Ly $\alpha$ data (Spergel et al. 2003).

Type-Ia SN and X-ray cluster data thus support a picture of a universe which is presently in a state of accelerated expansion, where NEC is most probably not violated (no superacceleration with a possible catastrophic ending, McInnes 2002), and in which a cosmological constant or something like it provides the dark energy.

However, one still has to be cautious with conclusions about NEC and SEC because they are only tested under restricted conditions. Furthermore, we are aware of the necessity to study in much more detail our assumptions that both galaxy clusters and SNe Ia do not evolve over the respective redshift ranges covered by the given observations. Moreover, important relations like the total cluster mass/X-ray luminosity relation and the extinction law relevant for nearby and distant SNe have to be known with much higher precision because the deviations from $w_{\mathrm{x}}=-1$ at $z=0$ for interesting dark energy scenarios might be smaller than the error bars of the present results. The present investigation tries to take into account possible systematic errors of the treatment of the X-ray clusters by using a quite large $\sigma_{8}$ marginalization interval. Future measurements based on improved relations can work with smaller intervals expected to provide quite precise cosmological constraints on both $\Omega_{\mathrm{m}}$ and $w_{\mathrm{x}}$.

Acknowledgements. We thank Stefan Gottlöber and Stefano Ettori for useful discussions. PS acknowledges financial support under grant No. 50 OR 0108. RRC thanks the Santa Barbara KITP for hospitality. This work was supported at the KITP by NSF PHY99-07949, and at Dartmouth by NSF PHY-0099543.

\section{References}

Allen, S. W., Fabian, A. C., Schmidt, R. W., \& Ebeling, H., 2002, MNRAS, submitted [astro-ph/0208394]

Amendola, L. 2000, Phys. Rev. D, 62, 043511

Baccigalupi, C., Balbi, A., Matarrese, S., Perrotta, F., \& Vittorio, N. 2002, Phys. Rev. D, 65, 063520

Bahcall, N. A., \& Fan, X. 1998, ApJ, 504, 1

Barceló, C., \& Visser, M. 1999, PhLB, 466, 127

Bond, J. R., Contaldi, C. R., Pogosyan, D., et al. 2002, AIP Conf. Proc., 646, submitted [astro-ph/0210007]

Böhringer, H., Guzzo, L., Collins, C. A., et al. 1998, The Messenger, 94, 21

Böhringer, H., Voges, W., Huchra, J. P., et al. 2000, ApJS, 129, 435

Böhringer, H., Schuecker, P., Guzzo, L., et al. 2001, A\&A, 369, 826

Böhringer, H., Collins, C. A., Guzzo, L., et al. 2002, ApJ, 566, 93

Borgani, S., \& Guzzo, L. 2001, Nature, 409, 39

Borgani, S., Rosati, P., Tozzi, P., et al. 2001, ApJ, 561, 13

Caldwell, R. R. 2002, PhLB, 545, 17

Caldwell, R. R., Dave, R., \& Steinhardt, P. J. 1998, Phys. Rev. Lett., 80,1582

Chiba, T., Okabe, T., \& Yamaguchi, M. 2000, Phys. Rev. D, 62, 023511

Carlberg, R. G., Yee, H. K. C., Ellingson, E., et al. 1996, ApJ, 462, 32

Collins, C. A., Guzzo, L., Böhringer, H., et al. 2000, MNRAS, 319, 939

Eisenstein, D. J., \& Hu, W. 1998, ApJ, 496, 605

Epstein, H., Glaser, V., \& Jaffe, A. 1965, NCim, 36, 2296

Ettori, S., Tozzi, P., \& Rosati, P. 2003, A\&A, 398, 879

Freedman, W. L., Madore, B. F., Gibson, B. K., et al. 2001, ApJ, 553, 47

Friedman, J. L., Schleich, K., \& Witt, D. M. 1993, Phys. Rev. Lett., 71,1486 
Flanagan, É.É., \& Wald, R. M. 1996, Phys. Rev. D, 54, 6233

Garnavich, P. M., Jha, S., Challis, P., et al. 1998, ApJ, 509, 74

Gromov, A., Baryshev, Yu., \& Teerikorpi, P. 2002, A\&A, submitted [astro-ph/0209458]

Guzzo, L., Böhringer, H., Schuecker, P., et al. 1999, The Messenger, 95,27

Haiman, Z., Mohr, J. J., \& Holder, G. P. 2001, ApJ, 553, 545

Hamuy, M., Phillips, M. M., Maza, J., et al. 1995, AJ, 109, 1

Hannestad, S., \& Mörtsell, E. 2002, Phys. Rev. D, 66, 063508

Hawking, S. W., \& Ellis, G. F. R. 1973, The large scale structure of space-time, Cambridge Monographs on Mathematical Physics, (London: Cambridge University Press)

Jenkins, A., Frenk, C. S., White, S. D. M., et al. 2001, MNRAS, 321, 372

Kaiser, N. 1984, ApJ, 284, L9

Lahav, O., Bridle, S. L., Percival, W. J., et al. 2002, MNRAS, 333, 961

Ma, C.-P., Caldwell, R. R., Bode, P., \& Wang, L. 1999, ApJ, 521, L1

Matarrese, S., Coles, P., Lucchin, F., \& Moscardini, L. 1997, MNRAS, 286, 115

Mayo, A. E., \& Bekenstein, J. D. 1996, Phys. Rev. D, 54, 5059

McInnes, B. 2002, JHEP, submitted [hep-th/0212014]

Melchiorri, A., Mersini, L., Odman, C. J., \& Trodden, M. 2002, submitted [astro-ph/0211522]

Morris, M. S., Thorne, K. S., \& Yurtsever, U. 1988, Phys. Rev. Lett., 61,1446

Moscardini, L., Matarrese, S., Lucchin, F., \& Rosati, P. 2000, MNRAS, 316, 283

Navarro, J. F., Frenk, C. S., \& White, S. D. M. 1997, ApJ, 490, 493

Netterfield, C. B., Ade, P. A. R., Bock, J. J., et al. 2002, ApJ, 571, 604

Peebles, P. J. E., \& Ratra, B. 2003, RMP, in press [astro-ph/0207347]

Penrose, R., \& Hawking, S. W. 1970, Proc. Roy. Soc. Lond., A 314, 529

Perlmutter, S., Pennypacker, C. R., Goldhaber, G., et al. 1995, ApJ, 440, L41

Perlmutter, S., Aldering, G., Goldhaber, G., et al. 1999, ApJ, 517, 565
Phillips, M. M. 1993, ApJ, 413, L105

Phillips, M. M., Lira, P., Suntzeff, N. B., et al. 1999, AJ, 118, 1766

Pierpaoli, E., Borgani, S., Scott, D., \& White, M. 2002, MNRAS, submitted [astro-ph/0210567]

Pryke, C., Halverson, N. W., Leitch, E. M., et al. 2002, ApJ, 568, 46

Ratra, B., \& Peebles, P. J. E. 1988, Phys. Rev. D, 37, 3406

Reiprich, T. H., \& Böhringer, H. 2002, ApJ, 567, 716

Riess, A. G., Filippenko, A. V., Challis, P., et al. 1998, AJ, 116, 1009

Rindler, W. 2001, Relativity: special, general, and cosmological (Oxford Univ. Press, Oxford)

Rosati, P., Borgani, S., \& Norman, G. 2002, ARA\&A, 40, 539

Schuecker, P., Böhringer, H., Guzzo, L., et al. 2001, A\&A, 368, 86

Schuecker, P., Guzzo, L., Collins, C. A., \& Böhringer, H. 2002, MNRAS, 335, 807

Schuecker, P., Böhringer, H., Collins, C. A., \& Guzzo, L. 2003, A\&A, 398,867

Scott, P. F. 2002, MNRAS, submitted [astro-ph/0205380]

Sievers, J. L., Bond, J. R., Cartwright, J. K., et al. 2002, ApJ, submitted [astro-ph/0205387]

Spergel, D. N., Verde, L., Peiris, H. V., et al. 2003, ApJ, submitted [astro-ph/0302209]

Stompor, R., Abroe, M., Ade, P., et al. 2001, ApJ, 561, L7

Szalay, A., Jain, B., Matsubara, T., et al. 2001, ApJ, submitted [astro-ph/0107419]

Torres, D. F. 2002, Phys. Rev. D, 66, 043522

Turner, M. S. 2002, in Hubble's Science Legacy: Future OpticalUltraviolet Astronomy from Space, ed. K. R. Sembach, J. C. Blades, G. D. Illingworth, \& R. C. Kennicut, ASP Conf. Ser., in press [astro-ph/0207297]

Visser, M. 1997, Phys. Rev. D, 56, 7578

Voges, W., Aschenbach, B., Boller, Th., et al. 1999, A\&A, 349, 389

Wald, R. M. 1984, General Relativity (Chicago and London: The University of Chicago Press)

Wang, L., \& Steinhardt, P. J. 1998, ApJ, 508, 483

Wetterich, C. 1988, NuPhB, 302, 668 